\title{
A Teaching Reform and Practice of Small-Class System in Optional Courses of Engineering Postgraduates
}

\author{
Guangqiang Li \\ School of Marine Electrical Engineering, Dalian Maritime \\ University \\ Dalian, 116026, China
}

\author{
Fengqiang Zhao \\ College of Mechanical \& Electronic Engineering, Dalian \\ Minzu University \\ Dalian, 116600, China
}

\begin{abstract}
Graduate education is the highest level of higher education. With the rapid development of economy and society in China, the scale of national graduate education is also expanding. The optional courses for engineering postgraduates are highly practical, and also there are usually many curriculums in major cultivation programs for engineering disciplines, resulting in the scattered graduates in various optional courses and the small teaching scale. In view of these characteristics, we take Modern Optimization Technology and Its Application, one optional course of the discipline of Control Science and Engineering at Dalian Maritime University, as an example and put forward three teaching reform ideas in order to improve the overall teaching quality. First, based on the small-class teaching methods, we elaborate the transformation of classroom teaching from traditional single subject of instructors to the double subjects of instructors and students, featuring weak-center distributed control teaching mode; Second, based on the scientific research project undertaken by instructors and combined with the port and shipping characteristics of Dalian Maritime University, we illustrate the research-and-discussion teaching of combining theory with practice, featuring guidance, learning, research and practice. The third is to put forward the diversified course assessments and closed-loop quality control system which are suitable for small-class teaching. After three years of practice, through questionnaires and final assessments, we can know that the overall teaching quality of this course is satisfactory, and the relevant methods and strategies can be widely applied to the teaching practice of other graduate courses.
\end{abstract}

Keywords-teaching reform; small-class teaching; teaching evaluation; optional courses; postgraduates

\section{INTRODUCTION}

At present, with the rapid development of China's economy and society, the demand for high-quality talents is increasing day by day, which promotes the development and improvement of national graduate education. Outline of the National Medium and Long-term Talent Development Plan (2010-2020) and Long-term Reform and Development Plan for National Education (2010-2020) point out that colleges and universities should take reform and innovation as the driving force and cultivate high-quality innovative talents to meet the needs of economy and society [1-2].

President Xi Jinping pointed out at the celebration of the 100th anniversary of the founding of the European and

Sponsored by the Graduate Education and Teaching Reform Project of Dalian Maritime University (YJG2019202).
American Alumni Association: "Talents are an important indicator of a country's comprehensive national strength. Only by realizing high-quality education can we cultivate more high-skilled, innovative and advanced talents, seizing the commanding height of talents competition, wining the initiative and the future." Premier Li Keqiang also pointed out: "We must persist in taking reform and opening-up as the driving force to speed up innovation and development with the support of human resources." Graduate education is the high end of China's higher education and directly responsible for the cutting-edge goal of high-level talents training. How to cultivate innovative and entrepreneurial talents to meet the needs of economy and society in large quantities has become an urgent task for graduate education.

After the expansion of undergraduate enrollments at the end of the last century and the rapid development of the scale of graduate education since this century, China has become the largest country of higher education in the world in terms of the number of students in colleges and universities, and the students' educational scale and level have jumped to a new stage. However, this trend brings some new problems to teaching, such as the shortage of instructors, classrooms and experimental equipment, etc., resulting in large classes in most colleges and universities.

But large-class teaching emphasizes the common development of students while it neglects the growth of individual personality, resulting in students' lack of the breadth of discipline knowledge, critical thinking and the courage to challenge authority. In order to cultivate students' critical thinking in graduate education, it is necessary to change teaching methods from traditional education to heuristic teaching, critical discussions and examinations with non-standard answers.

Compared with the traditional way of "infusion education" in large-class teaching, small-class teaching [3-4] has the advantages of easy teaching managements and convenient interactions between instructors and each student, giving full play to students' inspiration and creativity. On the other hand, with the development of science and technology, for engineering graduate students, new courses in major cultivation programs are emerging day by day, resulting in the dispersion of optional courses in most engineering majors and the small teaching scale, which is just suitable for the small-class system to organize teaching and conducive to creating more graduate courses with innovative thinking and heuristic education. 
Modern Optimization Technology and its Application is an optional course for graduate students in Control Science and Engineering of Dalian Maritime University with 36 hours and 2 credits. It mainly describes the basic theories of optimization technology, classical and intelligent optimization algorithms and its application in related engineering fields. The average number of students choosing this course in the past three years is 9 per year, which makes it suitable for small-class teaching. Therefore, taking the course-teaching as the background, it is of significance to explore the small-class teaching model that is suitable for the real national and school conditions of graduate students. Related researches and works can also be applied to the teaching of other graduate courses.

\section{An Outline of SMall-Class Teaching AT Home AND ABROAD}

According to the number of students in them, the classes can be divided into large ones and small ones. At present, there is no unified statement on the definition of a small class in terms of number of students. It is generally believed that the class with less than 30 students is a small class and the one with more than 30 students is regarded as a large class. In view of easy teaching management and teaching methods reform, the educational circles at home and abroad have carried out considerable explorations and researches in small-class teaching in higher education [5-6].

The small-class teaching in British colleges and universities has a long history [7]. For example, London School of Economics (LSE) stipulates that all classes cannot exceed 15 students. If the number of students is exceeded, classes must be split to more ones. The School of Oriental and African Studies at the University of London (SOAS) carries out the standard that the number of students in each class should not exceed 16 and 18 is the upper limit. At Oxford and Cambridge University, the upper limit is 12 , beyond which classes must be split to more ones. Furthermore, the United Kingdom has developed a set of teaching evaluation system for small-class teaching in colleges and universities. How well a school evaluates its teaching has a direct impact on its outcome in the school star rating published annually by the Council of Higher Education of UK.

The evaluation of small-class teaching in higher education of UK is mainly divided into the following three phases. The first phase of evaluation is arranged at the end of the first semester (before Christmas) mainly by students' online evaluation. The purpose is to obtain the students' feedback as soon as possible so as to discover the problems and help instructors to sum up the teaching. In the second phase, the Peer Observation is carried out in the second and third semesters, which is mainly conducted by the professional appraisers of Teaching Center by sitting in the classrooms to observe the teaching. The role of this Peer Observation is to make up for the bias that may be caused by online testing. Finally, when the year-end test results come out, the Teaching Center will also invite instructors to do a test and have a talk concerning the performances of students in order to improve the quality of teaching.

In the United States, although large classes are common in comprehensive research universities, many of which still offer small classes with high quality [8]. According to the US News report on undergraduate programs in American universities, the number of small and medium-sized classes in 265 comprehensive universities accounts for $45 \%$, while the proportion in Colleges of Arts and Sciences is as high as 65.2\%. In particular, several top American universities have a higher proportion of small classes, namely Harvard University (79.5\%), Columbia University $(79.7 \%)$ and Yale University $(75.8 \%)$.

At present, some domestic universities such as Tsinghua University, Peking University, Sichuan University and Dalian University of Technology, etc., are carrying out the reform and implementation of small-class teaching to a certain extent [9]. In the fall semester of 2012, Peking University launched a pilot project of small-class teaching in five departments as an important attempt to improve the quality of undergraduate talents cultivating. Sichuan University adopts large-class teaching \& small-class discussion, combined with the project of Training Young Teachers by Senior Instructors and Employing Graduates be Teaching Assistants. As early as 2006 the Material Processing Course of Tsinghua University changed the way of teaching from a large class (100 to 200 students of a same school) to teach in a small class (20 to 30 students) via case-based and discussion-based teaching modes, aiming to deepen students' understanding of technology and stimulate students' interests in learning. The Advanced Mathematics, a course of Dalian University of Technology also adopts large-class teaching and small-class tutoring. The instructors are teaching in large classes while the Ph.D. candidates are arranged as teaching assistants to be responsible for exercises classes, extracurricular tutorials and answering questions from students, which not only improves the teaching effects, but also gives the doctoral students the opportunities to participate in teaching.

To sum up, small-class teaching can inspire students creative consciousness and conveniently organize and manage teaching, which has been increasingly widely studied and applied in the field of higher education at home and abroad. Graduate courses, optional courses of engineering graduate students in particular, have their own characteristics of strong practicality, scattered number of students and so on, which are suitable for small-class teaching. Based on Modern Optimization Technology and its Application, this paper studies some problems in the teaching reform of small-class teaching in this course.

\section{TEACHING METHOD REFORM OF THE COURSE}

Small-class teaching doesn't merely refer to the size of classes, but also refers to the reform of teaching methods, teaching ideas and the teaching design of the courses. Compared with the traditional teaching methods, the small-class teaching models can effectively combine classroom teaching \& discussion, after-class materials-searching \& self-study, scientific research and engineering practice.

After discussion among students, students and instructors analyze and explore the ways to solve various problems to help students acquired knowledge, obtain experience and master the basic principles of modern optimization technology more deeply. The specific contents of this course reform are mainly reflected in the following measures.

\section{A. Transformation from classroom teaching to double-subject teaching method}

In view of the fact that graduate students already have mastered the basis of professional theoretical knowledge and the main contents of this course are both generality and individuality, as well as engineering application of 
optimization algorithms, which is especially suitable for students in small classes to present and discuss in groups and master the course contents actively via self-learning, team learning, mutual discussion between instructors and students. Only in this way can we successfully transfer the class into common protagonists of instructors and students so as to achieve the purpose of cultivating students' research-oriented learning abilities.

Specifically, in the class organization, instructors teach introduction, basic mathematical knowledge, and one-dimensional searching methods at first, and then list several classical and modern algorithms for students to choose according to their own interests and knowledge structures. As for the complex algorithms, several students can form a team to consult the literatures, conduct research via self-learning \& cooperative learning, as well as face-to-face discussion and interaction with instructors by QQ, WeChat, etc. in their spare time. Then, students are required to make PPTs that illustrate the basic principles, implementation methods, typical applications of these algorithms and present them in classes. After the presentation, an interval of time will be set aside for discussion among students followed by a designated time for instructors to give concluding guidance and answer students' questions. Finally, the Peer Review among students is adopted to allow them to evaluate and score the presentations of each student or team.

At the end of this course, a number of paper titles are listed for students to choose, which requires them to consult the literatures, search materials, collect data, analyze problems, program software and submit papers after the end of this course, giving full play to the advantages of small-class teaching and successfully making students the real protagonist in the teaching, which is exhibited in figure 1.

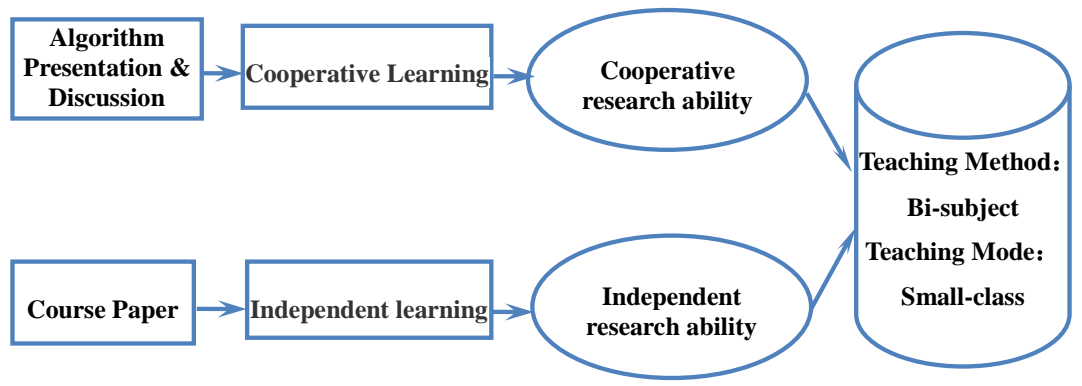

Fig. 1 Dual-subject diagram of teaching based on small-class teaching

\section{B. Teaching Methods Based on Combination of Research Projects and Port \& Shipping Characteristics}

Course instructors have been engaged in research on intelligent systems, optimization of intelligent systems and automated container terminals for many years. Combined with these research interests and National Natural Science Foundation of China (No. 61374114, 51809028) undertaken by the instructors, research-and-discussion teaching is carried out with the characteristics of guidance, self-learning, research and practice. Also the application of algorithms in the complicated engineering projects in the port and shipping field with characteristics of Dalian Maritime University is strengthened, aiming to improve the interests and participation of graduate students in teaching and facilitate their understanding of theoretical knowledge and practical engineering.

As an optional course for graduate students in Control Science and Engineering, this course theoretically discusses various classical climbing-hill optimization algorithms, such as the Powell method, Variable Metric Method via using derivative information and intelligent optimization algorithms belonging to swarm intelligence, such as Genetic Algorithm, Ant Algorithm, Particle Swarm Optimization and so on. In practice, these algorithms have many applications and can be widely used to solve all kinds of complex engineering optimization problems.

As a result, combined with scientific research projects and the port \& shipping with characteristics of Dalian Maritime University, a special project on the application of engineering optimization is designed in the course to organize students to consult materials on their own, which can help students be familiar with the engineering background of practical problems, e.g. ship dynamic positioning, intelligent stowage of automatic container terminal, AGV path planning, gantry crane scheduling, storage yard allocation and so on. And students can discuss the application of related algorithms in these engineering projects, such as the establishment of optimal mathematical model, the selection of algorithms, the design of coding and operators, the processing of result data and so on.

The related problems can be designed as software and programming homework with actual working conditions and parameters for students to program, which can be recorded as part of the course evaluation. It not only helps students to utilize the theoretical optimization algorithms in practice, but also further improves students' ability to make good use of this professional expertise to solve real engineering problems and stimulates students' interests in autonomous learning.

\section{Diversified Assessments and Closed-loop Teaching Quality Control System}

The small-class teaching mode and effects of the dual subjects of instructors and students need to be verified by a fair and objective course assessment and evaluation system, which can help students spend efforts during the whole semester without just cramming for final examination.

After the teaching reform, based on course papers (about $30 \%$ of the total score), PPT presentation and discussion of algorithms in class (about $30 \%$ of the total score), software system and programming for solving practical engineering problems (about $40 \%$ of the total score), the students' academic achievements are comprehensively evaluated, which put an end to rote memorization and encourage students' free exploration. It is advocated that students should utilize basic theoretical knowledge to analyze and solve practical engineering optimization problems. 
Specifically, the quality of course papers is mainly evaluated by instructors from the aspects of innovation, content quality, writing attitudes and the format. The presentation of PPT is evaluated by Delphi Method [10], which forms the evaluation consisting of the instructor's evaluation (weight $60 \%$ ), students' self-evaluation (weight 20\%) and classmate mutual evaluation (weight 20\%). As for the part of software system and programming, the programming language is not limited to a certain one. While the program frame, data structure, readability and maneuverability, calculation efficiency and correctness of calculation results are adopted and set its full sore for each item respectively. Subsequently, the instructors give every item a score for each student, and finally calculate the average score as the final score of a student.

As for scoring PPT presentation, the instructors mainly evaluate the students' preparation, oral presentation and subsequent algorithm discussion. Students' self-evaluation and peer evaluation are measured by the designed rating form, which focuses on four major items, namely the students' ability to ask questions, the ability to solve problems, the ability to work as a team and the ability to express. And a number of secondary indicators are designed for each of the above four items.

Through the above statement, students' final scores are given as far as objective, fair and diversified. The average scores of all the above items and goal achievement degree make up students' final scores, which gives the instructors and the research group a final result.

Finally, at the end of each course, instructors draw up a questionnaire and conduct a survey on graduate students either by directly handing them out in class, or via WeChat and Email after class. And the instructors will interview with some representative students as well. The statistics of the above questionnaire, feedback information of interviews and the final exam results are all submitted to instructors and research group to form a closed-loop teaching quality control system to promote the continuous improvement of teaching, which is exhibited in figure 2 .

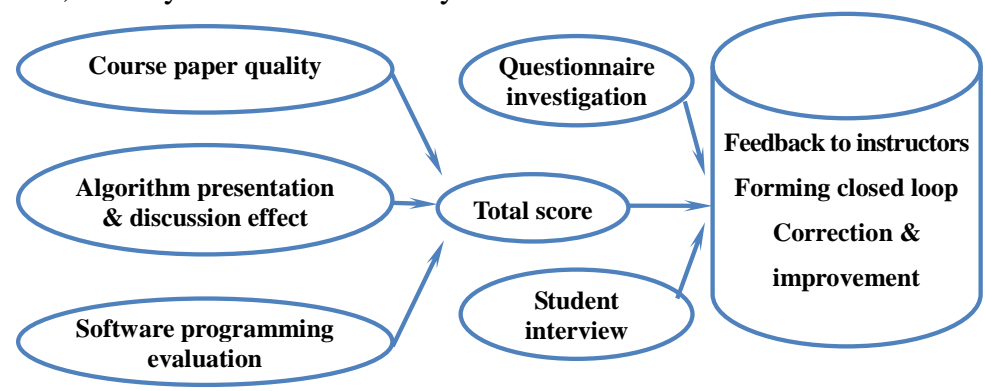

Fig. 2 Diversified evaluation methods and closed-loop quality control

\section{CONCLUSION}

Under the guidance of teaching reform mentioned above, instructors have revised syllabus, amended teaching objectives, diversified assessment \& evaluation methods, and designed a feedback questionnaire to investigate the graduate students who have taken this optional course. In the past three years, i.e. from 2017 to 2019, a total of 27 graduate students in discipline of Control Science and Engineering at Dalian Maritime University have taken this course. Among them, 9 were excellent ( 90 points or above) accounting for $33.3 \%, 13$ were good (80 to 90 ) accounting for $48.2 \%$, and 5 were average ( 70 to 80 ) accounting for $18.5 \%$, and nobody failed or scored below 70. In addition, according the past three years' questionnaires, the proportion of students, who were "very satisfied" or "satisfied" with the course, were $78.3 \%$ (2017), $80.5 \%$ (2018) and $81.8 \%$ (2019) respectively. None of the students choose "dissatisfied" and the rest of them choose "basically satisfied". One graduate student chosen this course, whose dissertation entitled "Research on Robot Path Planning Problems Based on Improved Fruit Fly Optimization Algorithm", won the National Scholarship for Postgraduates in 2018.

In conclusion, the distribution of the final scores of students, the feedback of the questionnaire and the award-winning of the student manifest that the teaching reform measures have made some achievements in practice. The relevant measures and methods can be widely applied to other optional courses, which are also expected to provide inspiration and reference for teaching reform of other compulsory courses of engineering graduate students.

\section{REFERENCES}

[1] R. Sun. "Progress Evaluation and Outlook of Talent Policy since the Implementation of the Outline of the National Medium and Long-term Talent Development Plan", Chinese Personnel Science, no.7, pp. 61-71, 2018.

[2] H. Liu. "Research on the Process of Educational Policy Making: A Case Study of the Process of Long-term Reform and Development Plan for National Education (2010-2020)", Higher Education Review, vol. 5, no.1, pp. 212-223, 2017

[3] P. Tang, X. Zhu, Y. Gao, et al. "Practice on Inquiry-based Small-Class Teaching Mode", Experiment Science and Technology, vol. 15, no. 4, pp. $68-71,2017$.

[4] C. Chen, J. Niu. "Comparing Effects of Small-Class Teaching and Large-Class Teaching on University Students' Math Achievement", Journal of Mathematics Education, vol. 26, no. 5, pp. 93-98, 2017.

[5] A. M. Alvero, K. Mangiapanello, J. Valad. "The effects of incentives, instructor motivation and feedback strategies on faculty evaluation response rates in large and small class sizes", Assessment \& Evaluation in Higher Education, vol. 44, no. 4, pp.501-515, 2019.

[6] S. Qin, Z. Zhang, Z. Jiao. "A Probe into Model of Lecture in Large Class \& Seminar in Small Class Based on Public Basic Course in Colleges and Universities", Journal of Hangzhou Dianzi University (Social Science Edition), vol. 15, no.1, pp. 65-69, 2019.

[7] X. Liu, X. Lin, X. Li. "Small class teaching in British higher educational institutions", Journal of Hebei Normal University (Educational Science Edition), vol. 11, no. 12, pp. 108-112, 2009.

[8] H. Zhang. "The Scale and the Achievements of University-An Empirical Study Based on USNEWS American University Rankings", Studies in Foreign Education, vol. 45, no. 331, pp. 55-66, 2018.

[9] P. Wang, X. Yang, W. Li, et al. "Discussion on small class teaching mode reform at colleges and universities", Education Teaching Forum, no. 14 , pp. 96-97, 2015

[10] S. E. Seker. "Computerized argument Delphi technique", IEEE Access, vol. 3, pp. 368-380, 2015. 